\title{
EVOLVED DUAL WEIGHT NEURAL ARCHITECTURES TO FACILITATE INCREMENTAL LEARNING
}

\author{
John A. Bullinaria \\ School of Computer Science, University of Birmingham, Birmingham, B15 2TT, UK \\ j.a.bullinaria@cs.bham.ac.uk
}

\begin{abstract}
Keywords: Incremental Learning, Evolutionary Computing, Neural Networks, Dual Weight Architectures.
Abstract: This paper explores techniques for improving incremental learning performance for generalization tasks. The idea is to generalize well from past input-output mappings that become available in batches over time, without the need to store past batches. Standard connectionist systems have previously been optimized for this problem using an evolutionary computation approach. Here that approach is explored more generally and rigorously, and dual weight architectures are incorporated into the evolutionary neural network approach and shown to result in improved performance over existing incremental learning systems.
\end{abstract}

\section{INTRODUCTION}

Learning from past input-output mappings in a way that generalizes well to produce appropriate outputs for inputs that have not been encountered before is a ubiquitous machine learning problem. Artificial neural network approaches have been particularly successful at this. However, there are critical limitations to this idea when the training data becomes available in batches over a period of time, such as the necessity to store past data, and the problem of incorporating the new data without having to start the training from scratch. For many real world applications, the learning really is an ongoing process, and efficient processes for using new data to improve performance are required (Giraud-Carrier, 2000). This is the problem of "incremental learning" that this paper addresses.

Past neural network approaches have been primarily concerned with memory tasks, rather than generalization tasks, and have tended to focus on minimizing catastrophic forgetting (Frean \& Robins, 1999; French, 1999). Progress there has been made by abstracting the complementary learning systems known to exist in the human brain (e.g., McClelland, McNaughton \& O'Reilly, 1995) to produce a range of successful coupled neural network systems (e.g., Ans et al., 2002). However, a much simpler approach based on optimizing traditional neural networks (Bishop, 1995) using evolutionary computation techniques (Bullinaria, 2007; Yao,
1999) has also produced improved performance (Seipone \& Bullinaria, 2005a). Moreover, combining previously studied dual weight architectures (Hinton \& Plaut, 1987) with the evolutionary approach can result in even more improvement (Seipone \& Bullinaria, 2005a).

For the kinds of generalization tasks more relevant to real world applications, some success has been achieved with the Learn ++ neural network ensemble approach of Polikar et al. (2001, 2002), but extending the evolutionary approach from memory tasks (Seipone \& Bullinaria, 2005a) appears to provide even better performance (Seipone \& Bullinaria, 2005b), though a more statistically rigorous study is needed to confirm that. The main aims of this paper are to test more carefully the basic evolutionary approach on generalization tasks, and to explore whether the introduction of dual weight architectures can lead to further improvements in the way they did for memory tasks.

The next section looks in more detail at incremental learning for generalization tasks, and Section 3 provides some baseline performance levels for a standard benchmark data-set. Then Section 4 describes how evolutionary computation techniques can be used to optimize neural systems. Section 5 presents results from a series of experiments that study the evolved incremental learning neural networks, and investigate whether dual weight neural architectures can result in further performance improvements. The paper ends with some conclusions in Section 6. 


\section{INCREMENTAL LEARNING}

The topic of interest here is generalizing well from past input-output mappings that become available in batches over time, without the need to store past batches. For such incremental learning systems to be considered successful, there are four crucial properties that are required (Polikar et al., 2001). Learning new data must:

1. result in improved generalization performance,

2. not require access to the previous training data,

3. not cause large scale forgetting of previously learned data, and

4. allow the accommodation of new data classes.

Humans seem to have all these properties, but achieving them in artificial neural network systems seems more difficult. When a neural network is trained on a new batch of data, its weights, which encode its input-output mapping, are modified. If this is not done carefully, the information learned from previous training patterns can be lost, and the performance can become worse rather than better. The aim is to update the weights in such a way that the new information is incorporated usefully, without excessively disrupting what was there before. One obvious approach, that only partially violates property 2 , is to keep a representative subset of the past training data, or pseudo data items that represent the past data, and use them along with the current data batch (Frean \& Robins, 1999).

Another approach, that has worked well for memory tasks, without violating property 2 , has been to employ dual neural networks (modeled on human brain regions) to interleave the new information with the old (e.g., Ans et al., 2002; McClelland, et al., 1995). A further approach has used simulated evolution by natural selection to generate standard neural networks with parameters that allow improved performance (Seipone \& Bullinaria, 2005a), and then this can be extended to evolve more sophisticated dual-weight networks, which have two additive weights between each pair of nodes, as originally proposed by Hinton \& Plaut (1987). There is one standard set of connection weights, and an additional set of "fast weights" that have larger learning rates and a large weight decay rate that prevents them from having long term memory. The additional set of weights allows the new information to be incorporated more smoothly, as with the dual network approach, but with a much simpler architecture (Seipone \& Bullinaria, 2005a).

For generalization tasks, most success was first achieved by somewhat different approaches. In particular, the Learn ++ algorithm of Polikar et al. (2001) employed an ensemble of weak classifiers to generate multiple hypotheses using training data sampled according to some tailored distributions. Their simulation results on a range of benchmark classification problems showed how this algorithm satisfied all the incremental learning properties listed above. However, this algorithm involves a large number of parameters that are fixed by hand in a rather ad hoc manner, and it has since been suggested that better results may be achieved using evolutionary computation techniques to optimize standard neural networks in the same way as for memory tasks (Seipone \& Bullinaria, 2005b).

\section{BASELINE PERFORMANCE}

Before exploring the novel approaches of this paper, it is useful to establish some baseline performance levels for traditional neural networks (Bishop, 1995) and for the Learn++ approach of Polikar et al. (2001, 2002). For concreteness, one particular standard classification problem will be studied, but the applicability to other problems should be clear. To facilitate comparison with the earlier research, the main incremental learning data set studied by Polikar et al. (2001, 2002) was chosen, namely the optical digits database from the UCI machine learning repository (Blake \& Merz, 1998). This contains hand-written samples of the digits 0 to 9 digitised on to an $8 \times 8$ grid to create 64 input attributes for each sample, with a training set of 3823 patterns and a separate test set of 1797 patterns, and the ten classes spread fairly evenly over both sets.

To study basic incremental learning, the training set is divided randomly into six distinct batches of 200 patterns (each with 20 patterns from each digit class) for six stages of incremental training, plus a further distinct sub-set of 1423 patterns to be used as a validation set during the evolution. That leaves another six batches of 200 unseen training patterns to be used only after the whole evolutionary process has been completed, which ensures that the evolved neural networks only see new data and fully satisfy property 2. The aim is to maximise the final generalization performance after training, and the validation set is used to estimate that to provide a measure of fitness to drive the evolutionary process. The test set is not used until the whole evolutionary process is completed, at which point it is used to evaluate the incremental learning performance of the evolved networks using the unseen training patterns. 
Table 1: Incremental learning performance for traditional back-propagation neural networks on the basic Optical Digits data set of Polikar et al. (2001). Mean percentage rates of correct classification, with standard errors in brackets.

\begin{tabular}{|c|c|c|c|c|c|c|}
\hline & $\boldsymbol{T}_{\boldsymbol{1}}$ & $\boldsymbol{T}_{\mathbf{2}}$ & $\boldsymbol{T}_{3}$ & $\boldsymbol{T}_{4}$ & $\boldsymbol{T}_{\mathbf{5}}$ & $\boldsymbol{T}_{\boldsymbol{6}}$ \\
\hline $\boldsymbol{B}_{\boldsymbol{1}}$ & $100.00(0.00)$ & $95.51(0.05)$ & $93.88(0.06)$ & $92.85(0.06)$ & $91.59(0.09)$ & $89.98(0.11)$ \\
\hline $\boldsymbol{B}_{2}$ & -- & $100.00(0.00)$ & $94.66(0.06)$ & $93.08(0.07)$ & $91.78(0.08)$ & $90.01(0.11)$ \\
\hline $\boldsymbol{B}_{3}$ & -- & -- & $100.00(0.00)$ & $93.87(0.07)$ & $91.87(0.08)$ & $90.08(0.12)$ \\
\hline $\boldsymbol{B}_{4}$ & -- & -- & -- & $100.00(0.00)$ & $92.69(0.09)$ & $90.44(0.12)$ \\
\hline $\boldsymbol{B}_{5}$ & -- & -- & -- & -- & $99.94(0.04)$ & $91.08(0.12)$ \\
\hline $\boldsymbol{B}_{\boldsymbol{6}}$ & -- & -- & -- & -- & -- & $99.59(0.08)$ \\
\hline Test & $88.09(0.04)$ & $89.56(0.03)$ & $89.43(0.04)$ & $88.97(0.04)$ & $87.95(0.07)$ & $86.39(0.10)$ \\
\hline
\end{tabular}

Table 2: The Learn++ incremental learning performance obtained by Polikar et al. (2001) on the basic Optical Digits data set. Mean percentage rates of correct classification (standard errors were not published).

\begin{tabular}{|c|c|c|c|c|c|c|}
\hline & $\boldsymbol{T}_{\boldsymbol{1}}$ & $\boldsymbol{T}_{\boldsymbol{2}}$ & $\boldsymbol{T}_{\mathbf{3}}$ & $\boldsymbol{T}_{\boldsymbol{4}}$ & $\boldsymbol{T}_{\boldsymbol{5}}$ & $\boldsymbol{T}_{\boldsymbol{6}}$ \\
\hline $\boldsymbol{B}_{\boldsymbol{1}}$ & 94 & 94 & 94 & 93 & 93 & 93 \\
\hline $\boldsymbol{B}_{\boldsymbol{2}}$ & -- & 93.5 & 94 & 94 & 94 & 93 \\
\hline $\boldsymbol{B}_{3}$ & -- & -- & 95 & 94 & 94 & 94 \\
\hline $\boldsymbol{B}_{\boldsymbol{4}}$ & -- & -- & -- & 93.5 & 94 & 94 \\
\hline $\boldsymbol{B}_{\boldsymbol{5}}$ & -- & -- & -- & -- & 95 & 95 \\
\hline $\boldsymbol{B}_{\boldsymbol{6}}$ & -- & -- & -- & -- & -- & 95 \\
\hline $\boldsymbol{T}$ est & 82.0 & 84.7 & 89.7 & 91.7 & 92.2 & 92.7 \\
\hline
\end{tabular}

The incremental learning takes place over six training sessions $T_{i}$, during each of which only one batch $B_{i}$ of 200 patterns is used to train the network to some stopping criterion. The highest activated output unit for each input pattern then indicates the network's classification output. At the end of each session, the network is re-tested on all the training batches from the previous sessions to see how much interference has taken place, and also on the validation set to provide a measure of generalization ability. As more of the training batches are used, the generalization is expected to increase, demonstrating good incremental learning capability, but at the same time the performance on the previous data batches should not be seriously reduced.

To see the problems that arise with standard neural networks, the performance for a traditional Multi-Layer Perceptron (Bishop, 1995) with one hidden layer was established. The nature of the data fixes the number of input units to be 64 , and the number of output units to be 10 , one for each class. One hundred such networks with 100 hidden units were initialized with all their random weights drawn uniformly from the standard range $[-1,1]$, and trained for 5000 epochs per data set, with all the gradient descent (back-propagation) learning rates fixed at 0.02 (i.e. just below the maximum value that allows stable training for this network), with no special learning features such as training tolerances, weight decay or sigmoid prime offsets. The average performances of these standard networks are shown in Table 1 as percentages. The columns show the classification performances at the end of each of the six stages of training $T_{i}$, on the current data-batch $B_{i}$, all previous data-batches $B_{j<i}$, and the test set. The generalization (test set) performance does increase with the first two batches, but then starts falling again as the later batches are learned, presumably because of over-fitting of the training data. Moreover, as each data-batch is learned, the performance on the earlier batches is seen to deteriorate. This is a clear indication of poor incremental learning ability. The aim, of course, is to identify the simplest ways of going beyond these standard neural networks to achieve better learning performance.

The first reasonably successful neural network incremental learning system was Learn ++ from Polikar et al. (2001), which on the same optical digits data achieved the performance shown in Table 2. Although the initial training results start lower, they remain more steady as further training databatches are used, and there is a steady increase in generalization ability as more data is made available. The generalization performance finishes at $92.7 \%$, compared with only $86.4 \%$ for the baseline standard neural networks. The question to be explored next is whether further improvements are possible using evolution and/or improved neural architectures. 


\section{NEURO-EVOLUTION}

The idea of applying the principles of evolution by natural selection to optimize the performance of neural networks is now widely established (e.g., Yao, 1999; Bullinaria, 2007). A population of individual neural networks is maintained, each with a genotype that represents an appropriate set of innate parameters. For each generation of evolution, the "fittest" individuals are taken to be those exhibiting the best performance on their given task and selected as parents. Suitable crossover and mutation operators then create offspring from those parents to populate the next generation, and the process is repeated until the fitness of the population levels off. This approach can be used to select optimal network topologies, learning algorithms, transfer functions, connection weights, and any other relevant network parameters (Yao, 1999).

An important feature of evolving neural networks is that any parameter can be subjected to the evolutionary process, and it is possible for many parameters that interact in complex ways to be evolved simultaneously. This means that the crucial and extremely difficult task of setting the learning parameters can be left entirely to the evolutionary process, rather than having to be done by the human designer, and improved performance is usually achieved (Yao, 1999; Bullinaria, 2007). However, the application of evolution is not totally straightforward, because obtaining optimal networks relies on maintaining diversity in the populations, and the evolutionary process can easily become trapped in local optima. Having appropriate initial populations is important, as is identifying good representations, crossovers and mutations, but often one must simply run the simulations many times and discard any that have clearly failed to achieved their true potential (Bullinaria, 2007)

In this study, the underlying network architecture and learning algorithm will continue to be standard fully connected Multi-Layer Perceptrons with sigmoidal processing units and one hidden layer, trained by gradient descent weight updating (backpropagation) with the Cross Entropy error measure (Bishop, 1995). The aim is to evolve the various neural network topology and learning parameters to produce systems that are good incremental learners. The connection weights themselves will be adjusted, as in humans, by the lifetime learning algorithm with new data, rather than by being specified as evolved innate parameters by the evolutionary process.

The simulated evolution involves populations of individual neural networks, each learning their weights starting from random initial weights drawn from their own innately specified distributions. The process starts from an initial population with random innate parameters, and for every generation, each network goes through the incremental learning process and has its fitness (i.e. estimated final generalization performance) determined. The fittest half of the population are then copied into the next generation, and also randomly select a partner to produce one child, thus maintaining the population size. The offspring inherit innate characteristics (i.e. parameter values) from the corresponding ranges spanned by both parents, with random Gaussian mutations added to allow values outside that range (Bullinaria, 2007). For each new generation, all the networks, both copies and offspring, have new random initial weights and go through the whole incremental learning process. This is repeated for enough generations that no further improvements are evident, and the set of networks most likely to generalize well on the unseen data sets is obtained. The main design problem is deciding which innate parameters are worth including in the genotype.

For this particular study, the best results are most likely to be achieved if all the traditional neural network parameters are evolved simultaneously:

1. The number of hidden units $N_{\text {Hid }}$ subject to some appropriate problem specific maximum which is significantly more than the number required to learn the training data, but not so large as to unnecessarily slow down the computational simulations. For the optical digits data set this maximum was set at 100 .

2. The connectivity level between layers $\left(c_{I H}, c_{H O}\right)$, specified as the proportion of possible connections that are used by the network. The specific connections used are chosen randomly.

3. The gradient descent learning rates $\eta_{L}$, for which earlier studies have established that allowing different values for each of the four network components $L$ (input to hidden weights $I H$, hidden unit biases $H B$, hidden to output weights $H O$, and output unit biases $O B$ ) results in massively improved performance over having a single value for all of them as in traditional hand crafted networks (Bullinaria, 2007).

4. The random initial weight distribution for each network component $L$. There are several options for specifying these, but here simple lower and upper limits of uniform distributions $\left[-l_{L}, u_{L}\right]$ are used.

5. The sigmoid prime offset $o_{S P O}$ which prevents saturation and poor learning at the hidden layer (Bullinaria, 2007).

6. A weight decay regularization parameter $\lambda$ that 
can act to prevent over-fitting of noisy training data (Bishop, 1995).

7. The output error tolerance $t$ that determines when a particular output activation is deemed "correct", and a training tolerance $s$ specifying the fraction of training parameters that can be left unlearned. These also act to prevent overfitting of noisy training data.

8. For the extra set of weights in the dual weight networks, there is also another weight decay rate $\delta$ and a scale factor $\sigma$ that multiplies the main learning rates $\eta_{L}$ (Hinton \& Plaut, 1987).

All the evolutionary simulations used populations of 100 networks, and the initial populations were created with random innate parameters drawn from traditional ranges (numbers of hidden units $N_{\text {Hid }}$ from $[0,100]$; learning rates $\eta_{L}$, initial weight distribution parameters $l_{L}, u_{L}$ and connectivity proportions $c_{I H}, c_{H O}$ from $[0,1]$; sigmoid prime offsets $o_{S P O}$ from $[0,0.2]$; standard weight decay parameters $\lambda$ from [0, 0.001]; tolerances $t$ from $[0,0.5]$; tolerances $s$ from $[0,1.0]$; dual weight decay parameters $\delta$ from $[0,0.2]$; and dual weight scale factors $\sigma$ from $[2,20])$. The precise starting parameter ranges had very little effect on the final results, but poor values often led to extremely slow starts to the evolutionary process. Then, for each new generation, each individual network had new random initial weights drawn from their own innately specified range, and learned according to their other innately specified parameters.

There were two obvious regimes for using the training data during the evolutionary process: new randomly selected training and validation sets as specified above could be selected for each new generation, or the same sets could be used for each generation. The idea here is to evolve systems that will work well on unseen data sets, and different training data for each generation proved to result in better general purpose learners, so this approach was adopted. For each network, each of the six training sessions continued until the innately specified tolerance was reached, or until a maximum number of training epochs was reached. That maximum number of epochs was set large enough that it only prevented successful learning during the first few generations when all the learning abilities were still very poor. At the end of each training batch, each network was re-tested on all the earlier training batches and the validation set. The individuals that had the lowest error on the validation set after training on all six data-batches were taken to be the fittest, and used to produce the next generation by copying and by using crossover and mutation.

\section{SIMULATION RESULTS}

To determine the best possible results that might be achievable from the training data, neural networks were first evolved to generalize as well as possible from only one batch of 200 patterns, and then from all six batches together (i.e. 1200 patterns) in a single training session. For both cases, exactly the same evolutionary regime was used as for evolving the incremental learners, with the best $10 \%$ of the evolved individuals on the validation set chosen for testing on unseen data. They were re-initialized and trained on new random training data-batches, and achieved average final test set performances of $91.55 \pm 0.03 \%$ for the 200 training patterns case, and $96.21 \pm 0.02 \%$ for 1200 patterns. This gave the best levels of performance that could reasonably be expected from the incremental learners.

Five independent runs of the evolutionary process were then carried out for the incremental learners, first for standard networks and then for networks with dual-weights (Hinton \& Plaut, 1987). The means and variances of the key parameters for the dual-weight case are shown in Figure 1. The top-left graph shows how the connectivity levels rise quickly to near full connectivity. The top-right graph shows how the learning rates $\eta_{L}$ evolve to fairly narrow bands, with most near the traditional values around 0.1 , but with the input-to-hidden learning rate some thousand times smaller. That pattern of learning rates would be very difficult to get right "by hand", but evolution finds it quite consistently. The middle-left graph shows how the sigmoid prime offset $o_{S P O}$ and standard weight decay parameters $\lambda$ fall to values so low that they have no significant effect on the performance of the networks. The middle-right graph shows how the fast weight decay rate $\delta$ settles at a higher level and, together with the fast weight scale factor $\sigma$, results in a system of dual weights with the required properties. The bottom-left graph shows how the training output tolerance $t$ and stopping early parameter $s$ evolve to appropriate values. Finally, the bottom-right graph shows how the generalization performance measures improve little after the first 600 generations, despite some of the parameters continuing to settle down towards their final values. The fluctuations in the parameters during evolution reflect how crucial each one is to the final fitness. The persistent fluctuations in the performance reflect the random nature of the training data sets and initial weight distributions. Not shown (due to lack of space) are the number of hidden units which quickly rises to near the maximum allowed, and the eight 

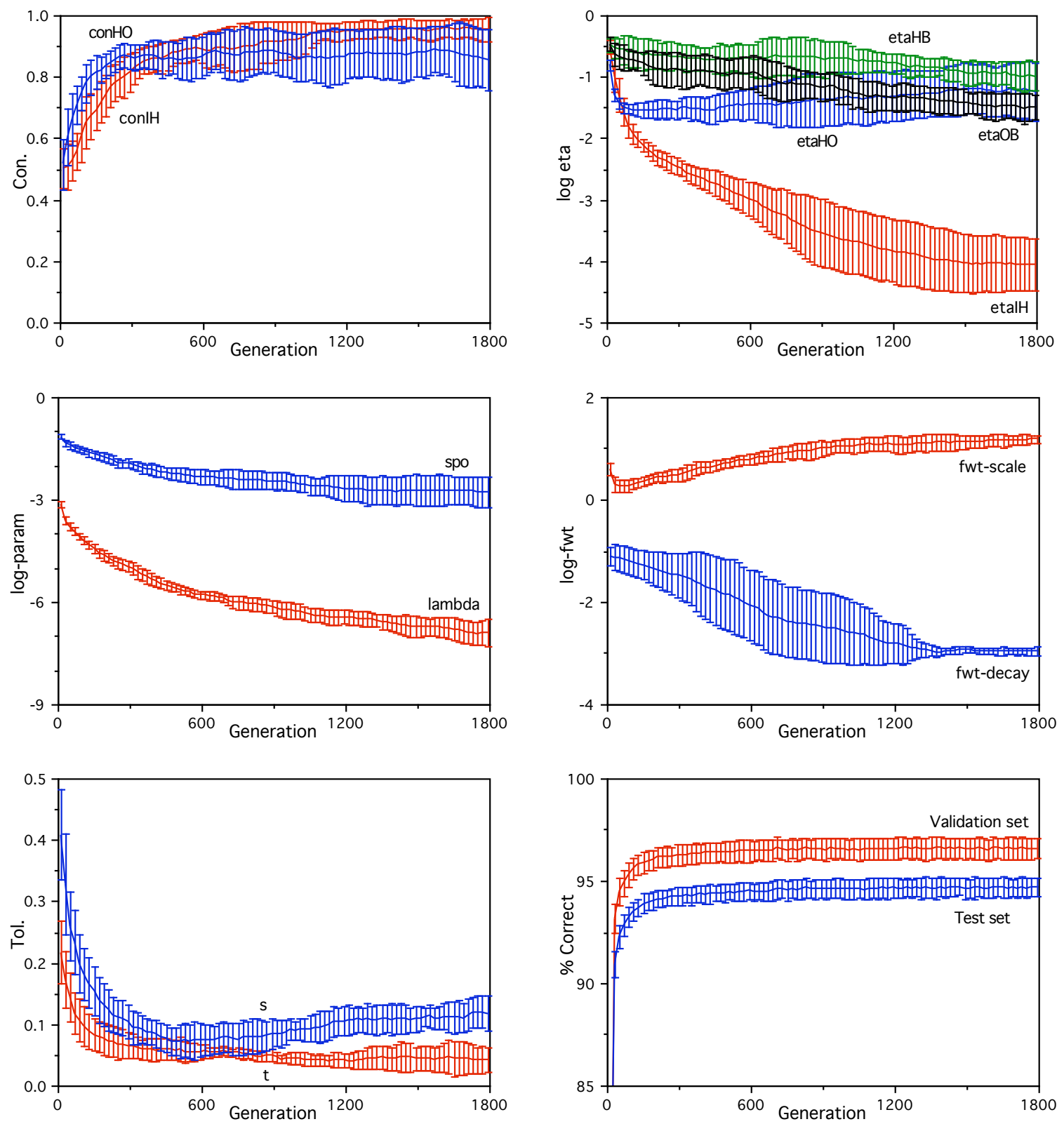

Figure 1: Evolution of the dual-weight neural network parameters and performance for the basic Optical Digits data set.

initial weight distribution parameters that exhibit a complex (though not particularly informative) pattern of appropriate values.

The population averages of Figure 1 are slightly misleading because high population diversity was deliberately maintained by mutations and crossovers to facilitate the evolutionary process, and that leaves many sub-optimal individuals in the population. A better performance indicator involves running just the best $10 \%$ of the final evolved populations (as measured on the validation set) many times and averaging. Then the variance across the means from the five evolutionary simulations gives the standard error on those means. Table 3 shows, in the same format as Table 1, the averages using 100 runs of each individual for the standard network case. There is a clear improvement in all aspects over the non-evolved baseline results of Table 1, and also over the Learn ++ results of Table 2. The performance levels on past training data batches still fall slightly as the later training batches are processed, but those performance levels remain well above those for Learn ++ . The generalization (i.e. test set) performance is also better than that of 
Table 3: Incremental learning performance of evolved neural networks without dual-weights on the basic Optical Digits data set. Mean percentage rates of correct classification, with standard errors in brackets.

\begin{tabular}{|c|c|c|c|c|c|c|}
\hline & $\boldsymbol{T}_{\boldsymbol{1}}$ & $\boldsymbol{T}_{2}$ & $\boldsymbol{T}_{3}$ & $\boldsymbol{T}_{4}$ & $\boldsymbol{T}_{5}$ & $\boldsymbol{T}_{\boldsymbol{6}}$ \\
\hline $\boldsymbol{B}_{\boldsymbol{1}}$ & $100.00(0.00)$ & $98.96(0.04)$ & $98.53(0.05)$ & $98.34(0.04)$ & $98.26(0.03)$ & $98.28(0.04)$ \\
\hline $\boldsymbol{B}_{2}$ & -- & $100.00(0.00)$ & $99.01(0.03)$ & $98.48(0.05)$ & $98.29(0.02)$ & $98.22(0.03)$ \\
\hline $\boldsymbol{B}_{3}$ & -- & -- & $100.00(0.00)$ & $99.07(0.05)$ & $98.55(0.05)$ & $98.37(0.04)$ \\
\hline $\boldsymbol{B}_{4}$ & -- & -- & -- & $100.00(0.00)$ & $99.11(0.05)$ & $98.61(0.03)$ \\
\hline $\boldsymbol{B}_{5}$ & -- & -- & -- & -- & $100.00(0.00)$ & $99.10(0.02)$ \\
\hline $\boldsymbol{B}_{6}$ & -- & -- & -- & -- & -- & $100.00(0.00)$ \\
\hline Test & $91.49(0.05)$ & $92.97(0.03)$ & $93.63(0.02)$ & $94.11(0.03)$ & $94.42(0.02)$ & $94.68(0.01)$ \\
\hline
\end{tabular}

Table 4: Incremental learning performance of evolved neural networks with dual-weights on the basic Optical Digits data set. Mean percentage rates of correct classification, with standard errors in brackets.

\begin{tabular}{|c|c|c|c|c|c|c|}
\hline & $\boldsymbol{T}_{\boldsymbol{1}}$ & $\boldsymbol{T}_{\mathbf{2}}$ & $\boldsymbol{T}_{\mathbf{3}}$ & $\boldsymbol{T}_{\boldsymbol{4}}$ & $\boldsymbol{T}_{\mathbf{5}}$ & $\boldsymbol{T}_{\boldsymbol{6}}$ \\
\hline $\boldsymbol{B}_{\boldsymbol{1}}$ & $100.00(0.00)$ & $99.17(0.06)$ & $98.96(0.09)$ & $98.87(0.07)$ & $98.83(0.06)$ & $98.83(0.06)$ \\
\hline $\boldsymbol{B}_{2}$ & -- & $99.99(0.01)$ & $99.28(0.02)$ & $98.99(0.02)$ & $98.85(0.04)$ & $98.80(0.04)$ \\
\hline $\boldsymbol{B}_{3}$ & -- & -- & $99.94(0.02)$ & $99.30(0.03)$ & $99.02(0.02)$ & $98.89(0.03)$ \\
\hline $\boldsymbol{B}_{4}$ & -- & -- & -- & $99.84(0.04)$ & $99.29(0.02)$ & $99.00(0.01)$ \\
\hline $\boldsymbol{B}_{5}$ & -- & -- & -- & -- & $99.74(0.06)$ & $99.23(0.04)$ \\
\hline $\boldsymbol{B}_{\boldsymbol{6}}$ & -- & -- & -- & -- & -- & $99.65(0.05)$ \\
\hline $\boldsymbol{T e s t}$ & $91.62(0.11)$ & $93.47(0.05)$ & $94.21(0.03)$ & $94.60(0.04)$ & $94.87(0.05)$ & $95.07(0.04)$ \\
\hline
\end{tabular}

Learn ++ at each stage, and shows a gradual improvement as more data batches are used, thus indicating good incremental learning. The final test set performance of $94.68 \%$ appears to be a small improvement over the Learn ++ value of $92.7 \%$, but it actually more than halves the gap between the incremental learning performance and the $96.21 \%$ obtainable by training on all the data at once. More importantly, the performance of $91.49 \%$ on just the first set of training data is now very close to the $91.55 \%$ obtained by networks evolved specifically to perform well on a single training data batch. By comparison, Learn ++ only achieves $82 \%$ after the first batch of data. This improvement can be very important for practical applications, as the trained system may have to be used at that early stage.

Introducing an additional set of dual weights can lead to significant performance improvements due to the ability to incorporate appropriate weight changes for new data patterns into the existing weights with minimal disruption (Seipone \& Bullinaria, 2005a). For the current application, the fast weights led to the improvements in performance seen in Table 4. Although the difference in final generalization performance from that of Table 3 is quite small, it is highly statistically significant (t-test $p=0.0005$ ). The improvements were also sufficient to drive the fast weight parameters to extremely consistent values across the five independent runs (scale factor $\sigma=17 \pm 2$, decay rate $\delta=0.0011 \pm 0.0002$ ), which again indicates the importance of this factor.

So far, the problem of accommodating changes in the data classes present in the batches of training data (i.e. incremental learning property 4 above) has not been addressed. Polikar et al. (2002) created a four-batch version of the optical digits data set that had different class instances missing from different batches, and showed how Learn ++ performed well on it. Table 5 compares the generalization (i.e. test set) performance on this for traditional neural networks, Learn++, evolved neural networks, and evolved neural networks with dual weights, using the same evolutionary process as described above. Here the evolved networks are not much better than Learn ++ , but introducing dual weights does lead to a large and statistically significant improvement (t-test $p=0.00007)$. In this case, the potential for new data interfering with what was previously learned is much greater than in the basic data set in which all the digit classes are equally represented in each data batch, so it is perhaps not surprising that the enhanced data interleaving provided by the dual weights is more helpful here.

Within this framework, there still remains scope for further improvement. As noted above, the evolutionary process makes full use of the maximum number of hidden units allowed, and it also tends to slow the learning to make full use of the maximum 
Table 5: Incremental learning performance for neural networks on the "varying classes" Optical Digits data set of Polikar et al. (2002). Mean percentage rates of correct test set classification, with standard errors in brackets.

\begin{tabular}{|c|c|c|c|c|}
\hline & $\boldsymbol{T}_{\boldsymbol{1}}$ & $\boldsymbol{T}_{\mathbf{2}}$ & $\boldsymbol{T}_{\mathbf{3}}$ & $\boldsymbol{T}_{\mathbf{4}}$ \\
\hline Traditional $\boldsymbol{N N}$ & $48.47(0.01)$ & $75.49(0.02)$ & $79.87(0.07)$ & $77.07(0.07)$ \\
\hline Learn++ & 46.6 & 68.9 & 82.0 & 87.0 \\
\hline Evolved $\boldsymbol{N N}$ & $48.59(0.09)$ & $76.39(0.18)$ & $84.78(0.47)$ & $87.41(0.39)$ \\
\hline Evolved NN with Dual-weights & $47.34(0.32)$ & $80.13(0.50)$ & $87.32(0.15)$ & $93.84(0.04)$ \\
\hline
\end{tabular}

number of epochs allowed. Both of these factors form part of the natural evolved regularization process, and further performance improvements may be possible by simply increasing those maximum values. However, the improvements achievable by doing this prove to be rather limited in relation to the enormous increase in the associated computational costs. Indeed, the computational cost of the general evolutionary process proposed in this paper is also high, but even small improvements are often extremely valuable, so it will generally remain a complex problem dependent decision whether the potential improvements are worth the extra effort.

\section{CONCLUSIONS}

This paper has provided a more general and more statistically rigorous confirmation of earlier results (Seipone \& Bullinaria, 2005b) indicating that the application of evolutionary computation techniques can massively improve the incremental learning abilities of standard neural networks on real-world generalization tasks compared to existing systems such as Learn++ (Polikar et al., 2001, 2002). It has also demonstrated that the same approach can be used to evolve more sophisticated dual-weight architectures that have further improvements in performance, particularly when the representation of classes varies between training data batches. Thus effective evolutionary neural network techniques have been established that can straightforwardly be tested on and applied to any future incremental learning problems requiring good generalization.

\section{REFERENCES}

Ans, B., Rousset, S, French, R.M., Musca, S., 2002. Preventing Catastrophic Interference in MultipleSequence Learning Using Coupled Reverberating Elman Networks. Proceedings of the Twenty-fourth Annual Conference of the Cognitive Science Society, 71-76. Mahwah, NJ: LEA

Bishop, C.M., 1995. Neural Networks for Pattern
Recognition. Oxford, UK: Oxford University Press.

Blake, C.L., Merz, C.J., 1998. UCI Repository of Machine Learning Databases. University of California, Irvine. http://www.ics.uci.edu/ mlearn/MLRepository.html

Bullinaria, J.A., 2007. Using Evolution to Improve Neural Network Learning: Pitfalls and Solutions. Neural Computing \& Applications, 16, 209-226.

Frean, M., Robins, A., 1999. Catastrophic Forgetting in Simple Neural Networks: An Analysis of the Pseudorehearsal Solution. Network: Computation in Neural Systems, 10, 227-236.

French, R.M., 1999. Catastrophic Forgetting in Connectionist Networks. Trends in Cognitive Sciences, 4, 128-135.

Giraud-Carrier, C., 2000. A Note on the Utility of Incremental Learning. AI Communications, 13, 215223.

Hinton, G.E., Plaut, D.C., 1987. Using Fast Weights to Deblur Old Memories. Proceedings of the Ninth Annual Conference of the Cognitive Science Society, 177-186. Hillsdale, NJ: LEA.

McClelland, J.L., McNaughton, B.L., O’Reilly, R.C., 1995. Why There Are Complementary Learning Systems in the Hippocampus and Neocortex: Insights From the Successes and Failures of Connectionist Models of Learning and Memory. Psychological Review, 102, 419-457.

Polikar, R., Byorick, J., Krause, S., Marino, A., Moreton, M., 2002. Learn++: A Classifier Independent Incremental Learning Algorithm for Supervised Neural Networks. Proceedings of the 2002 International Joint Conference on Neural Networks, 2, 1742-1747.

Polikar, R., Udpa, L., Udpa, S.S., Honavar, V., 2001. Learn++, 2001. An Incremental Learning Algorithm for Multi-Layer Perceptron Networks. IEEE Transactions on Systems, Man, and Cybernetics-Part C: Applications and Reviews, 31, 497-508.

Seipone, T., Bullinaria, J.A., 2005a. The Evolution of Minimal Catastrophic Forgetting in Neural Systems. Proceedings of the Twenty-Seventh Annual Conference of the Cognitive Science Society, 19911996. Mahwah, NJ: LEA.

Seipone, T., Bullinaria, J.A., 2005b. Evolving Improved Incremental Learning Schemes for Neural Network Systems. Proceedings of the 2005 IEEE Congress on Evolutionary Computing (CEC 2005), 273-280. Piscataway, NJ: IEEE.

Yao, X., 1999. Evolving Artificial Neural Networks. Proceedings of the IEEE, 87, 1423-1447. 